\title{
Phase effect in time-stamped accelerometer measurements - an experimental approach
}

\author{
F. Moschas and S. Stiros ${ }^{\star}$ \\ Laboratory of Geodesy and Geodetic Applications, Department of Civil Engineering, University of Patras, Greece
}

Received: 20 October 2012 / Accepted: 28 October 2012

\begin{abstract}
The phase error in a high quality accelerometer was investigated on the basis of systematic experiments on a prototype platform permitting controlled vertical harmonic oscillations. The recordings of an accelerometer and of an ultra-high frequency GPS fixed on this platform were compared with those of a LVDT transducer, controlling the platform movement. All sensors were supported by independent GPS timing. The output of this study is that the recordings of accelerometers are characterized by a random phase error, which, however, decreases with the increase of the oscillation frequency, as dictated from the laws of mechanics. Such errors are important given the increasing use of combinations of accelerometers with various sensors for a wide range of applications. A simple method for the correction of such errors is proposed.
\end{abstract}

Keywords: Accelerometer; LVDT; dynamic measurements; phase-shift; time-delay; GPS stamping; error

\section{Introduction}

In the last years or even decades, there have been introduced various measuring and recording instruments with relatively high sampling rates, though sometimes as low as $1 \mathrm{~Hz}$, a rate which is indeed fast for satellite positioning. The advent of such instruments opened new horizons in various types of measurements, for the additional reason that it permitted to combine different sensors and obtain combined observations. Some examples are (1) the time stamping of seismometers with GPS and the combination of seismometers or accelerometers with GPS [1], permitting a better understanding of earthquakes and structural vibrations; (2) the development of inertial systems consisting of gyroscopes and accelerographs which are combined with GPS and permit to counteract satellite signal deficiencies [2]; (3) the combination of GPS with sonars which permit the improvement of the reconstruction of the seabottom topography [3]; or finally (4) combination of various sensors to obtain 3-D images using mobile mapping techniques $[4,5]$. A key point in all these studies is the time stamping of observations which, at least in the last years, tends to be provided by GPS observations [6,7], avoiding older techniques such as simultaneous conventional (ana$\log$ ) photos of chronometers and of other analog sensors such as telescope images.

However, synchronization of different sensors is not without problems, and a time-shift between measurements of different instruments exists in many cases. The importance of this delay has been emphasized by
Hessling [8], who proposed its stochastic estimation on the basis of cross-correlation, before the dynamic error in measurement systems is computed.

In order to shed light to this problem, especially for accelerometers which are used in a wide range of applications (inertial positioning systems, structural monitoring, earthquake monitoring, etc.), we made systematic experiments using an accelerometer collocated with other instruments (GPS as displacement sensors, LVDT transducer, etc.), all with independent GPS time stamping, in order to examine the statistics and pattern of the possible phase between their recordings. The results of this study, focusing on the phase error of accelerometers, mostly derived from extensometer measurements, are summarized below.

\section{Methodological background}

We examine a harmonic oscillation of frequency $f$ described by equation (1)

$$
x(t)=X \cos (2 \pi f t-\varphi)
$$

which indicates that the instantaneous displacement $x(t)$ is a function of the amplitude $X$ of the oscillation, of time $t$ and of a phase $\varphi$. Derivation of this equation leads to equation (2) describing the instantaneous velocity $v(t)$

$$
\begin{aligned}
v(t) & =-2 \pi f X \sin (2 \pi f t-\varphi) \\
& =2 \pi f X \cos \left(2 \pi f t-\varphi-\frac{\pi}{2}\right)
\end{aligned}
$$


which is at phase $\pi / 2$ with the displacement. A second derivation leads to equation (3)

$$
\begin{aligned}
a(t) & =-(2 \pi f)^{2} X \sin \left(2 \pi f t-\varphi-\frac{\pi}{2}\right) \\
& =(2 \pi f)^{2} X \cos (2 \pi f t-\varphi-\pi)
\end{aligned}
$$

which indicates that in a harmonic oscillation the acceleration $a(t)$ is at phase $\pi$ (180 degrees) with displacement [9].

For this reason LVDT and accelerometer measurements in harmonic oscillations should ideally be at a phase of $\pi$ radians.

However, measurements of all instruments are affected by errors, and for this reason both the instantaneous amplitude and phase differ from the theoretical values.

In the present study measurements from the LVDT were used as a reference providing theoretical values in order to examine phase drifts in the accelerometer data. This selection was assessed by various experiments with different instruments as is further discussed in Section 5.

\section{Description of the experiments}

\subsection{Experimental setup}

For our experiments we used a prototype platform designed to produce vertical harmonic oscillations of controlled characteristics (amplitude/frequency) and to host various sensors. The platform permits to compare the output of various under-test sensors with certain reference sensors; hence it permits to define their accuracy, not simply their precision [10].

The experimental platform used consists of a horizontal plate sliding vertically with minimum friction on four vertical metal pylons fixed on a stable steel base. The latter is clamped on a concrete base. An electric motor with a DC stabilizer fixed on the steel base produces harmonic cyclic motions which are transformed to vertical linear oscillations using an adjustable mechanical eccentric converter of circular-to-linear motion (invertor) (Fig. 1).

The desired oscillation frequency is defined by a potentiometer, while the amplitude of the oscillation is defined by the adjustable invertor. The vertical movement (displacement) is recorded by a LVDT transducer, the two edges of which are fixed on the moving plate and the fixed base plate. The LVDT transducer is combined with a recorder with independent GPS time stamping.

Several under-test sensors can be installed on the moving platform and this gives the opportunity to compare their performance in recording the same motion, the characteristics of which are defined by the LVDT (reference sensor).

The prototype experimental platform was designed in the Geodesy Lab (Department of Civil Engineering, University of Patras) and was built in the Machine Shop of the Department of Physics (University of Patras). Construction of the experimental platform was constructed in the framework of the Diploma Thesis of Spyropoulos [11]. This device is different from others used in the past mostly

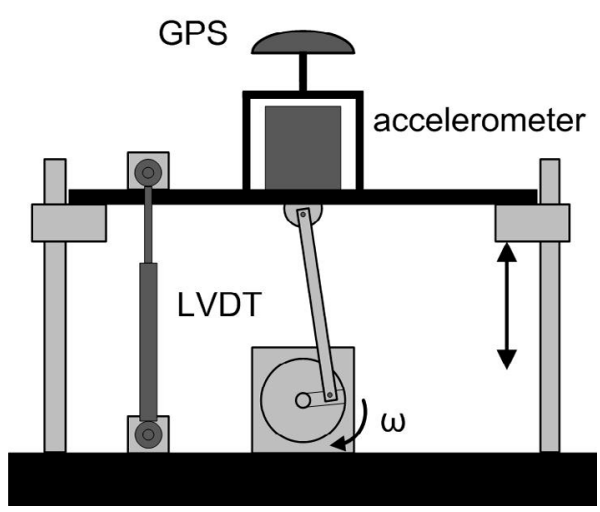

Fig. 1. Schematic side view of the prototype experimental platform used in the experiments. A double arrow indicates the sense of vertical harmonic oscillation of the horizontal platform (upper plate in black) on which tested sensors such as GPS and accelerometer are set. Oscillations are produced by conversion of the rotation generated by an electric motor (not shown) through a mechanical eccentric convertor and are recorded by the LVDT (reference sensor). Two of the metal pylons which are fixed on the horizontal platform base (lower horizontal plate in black) are shown. The horizontal platform hosting the test instruments is able to slide along the vertical pylons.

for the assessment of GPS measurements $[10,12]$ because it permits oscillations with pre-defined characteristics along the vertical axis, along which GPS presents the largest uncertainties [13], and because the motion of the platform is controlled by the LVDT sensor.

\subsection{Instruments and experimental procedure}

Experiments discussed in this paper were based on the comparison of LVDT reference recordings with recordings of a strong motion force-balance accelerometer and an ultra-high rate GPS unit. The LVDT and the accelerometer were connected to different recorders with different GPS timing unit, so that all three instruments were fully independent, each with different recording unit and timing. In addition, power supply in all instruments was provided by batteries in order to avoid noise from the electricity network.

In particular, we used a Geo-Sig AC-63 force-balance triaxial accelerometer sensor with a $200 \mathrm{~Hz}$ sampling rate (frequency range 0-100 Hz), a WayCon SL200-G-KR LVDT with range $\pm 100 \mathrm{~mm}$ or a WayCon SL100-G-KR LVDT with range $\pm 50 \mathrm{~mm}$, both with a sampling rate of $200 \mathrm{~Hz}$. 3-channel Geo-Sig GSR-18 recorders were used for the LVDT and the accelerometer, each with independent GPS timing sensor.

$100 \mathrm{~Hz}$ GPS units consisting of a Javad GrAnt antenna connected to a Javad Delta receiver were used, one on the platform of Figure 1 and another on a fixed nearby position (not shown in Fig. 1), in order to satisfy the requirements of the GPS kinematic positioning (movement of a moving antenna relative to a fixed antenna, DGPS). 
The experimental device and each one of the instruments were leveled before the experiment.

The experiments were made in four days (days 1 to 4 ), in the same place, and with the same hardware, with exception of the LVDT receivers: the SL200-G-KR sensor (range $\pm 100 \mathrm{~mm}$ ) was used in days 1 and 2, while sensor SL100-G-KR (range $\pm 50 \mathrm{~mm}$ ) was used in days 3 and 4 .

In total 86 oscillation events (20 events for day 1, 16 for day 2, 34 for day 3 and 16 for day 4 ) with an approximate duration of $70 \mathrm{~s}$ each were recorded and analyzed.

\section{Results}

After each experiment, data from the GSR-18 recorders were downloaded using the GeoDas $\AA$ software provided by GeoSig. During the preliminary analysis raw measurements from the LVDT were transformed into displacements (in millimeters) around the equilibrium point corresponding to zero, by subtracting the mean value for each event from the corresponding measurements. Acceleration time-series were provided in $\mathrm{g}$ units. Although we used tri-axial accelerometer, only the vertical component was recording a useful movement. GPS measurements were processed using the TRACK (kinematic module of GAMIT/GLOBK) software [14] and instantaneous coordinates of the oscillating GPS antenna in a local reference frame aligned with North, East and Up were obtained. Coordinates of the antenna were transformed into apparent displacements around zero (equilibrium point) using a linear transformation $[15,16]$. Typical time series from a set of recorded events during day 1 are presented in Figure 2.

The time interval of each oscillation event is the same in all instruments indicating that there are no large timeshifts between the instruments. At a first glance, LVDT and accelerometer measurements do not present significant noise, while GPS measurements are affected by long period noise with significant amplitude, a characteristic typical for GPS measurements [17-20].

From the total length of approximately $70 \mathrm{~s}$ of each event, the first and last five seconds were excluded from the analysis, because during these intervals the platform was accelerating or decelerating and the oscillation frequency was not constant ("transient oscillation" [10]).

For each of the 86 analyzed events, we produced diagrams showing the displacement and the acceleration as a function of time. A representative example is shown in Figures 3 and 4 . These diagrams indicated significant differences in the phases of the two variables, and this stimulated a further investigation.

\section{Statistical analysis of the phase differences}

The phase difference between the simultaneous recordings of the accelerometer and the LVDT (acceleration and displacement along the vertical axis) can be studied on the basis of cross-correlation or of spectral analysis. Clearly,

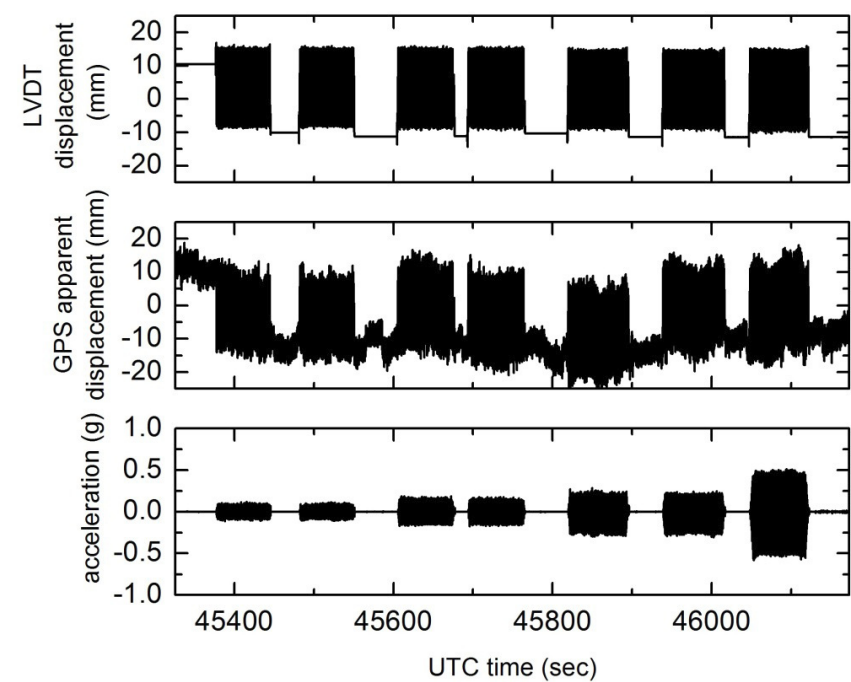

Fig. 2. Displacements derived from GPS and LVDT along with recorded accelerations. Data shown correspond to seven events during day 1.

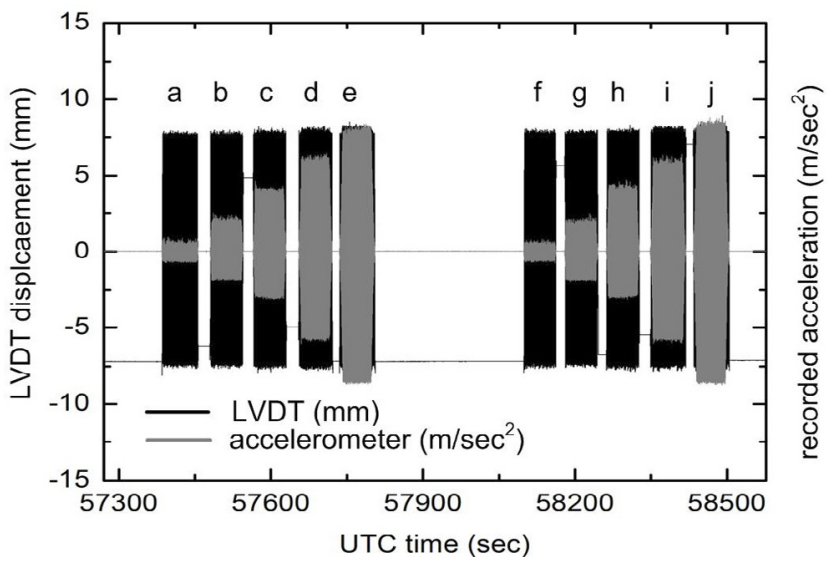

Fig. 3. Displacement and acceleration time history corresponding to 10 events $(\mathrm{a}-\mathrm{j})$ recorded in day 3 . All events are characterized by the same amplitude $( \pm 8 \mathrm{~mm})$. The oscillation frequencies are $1 \mathrm{~Hz}$ (events a, f), $2 \mathrm{~Hz}$ (events b, g), $3 \mathrm{~Hz}$ (events c, h), $4 \mathrm{~Hz}$ (events $\mathrm{d}, \mathrm{i}$ ) and $5 \mathrm{~Hz}$ (events e, j). The successive increase in frequency is depicted by the increase in maximum acceleration (grey line).

measurements are at the highest sampling frequency of the instruments, and hence the possibility of jitter noise cannot be ruled out [21]. Still, a preliminary analysis and comparison with the least-squares based on the NormPeriod software, which is ideal for non-equidistant data [22], revealed that results with FFT are satisfactory, given the length of the time series analyzed (approximate length of $60 \mathrm{~s}$ times $200 \mathrm{sps}=12000$ points). An additional test was made using data under-sampled at the $10 \mathrm{~Hz}$ rate, but results were similar.

In the present study we used the FFT function provided by Matlab ${ }^{\circledR}$ and for each of the 86 events we computed the spectrum and the phase for both the LVDT and 


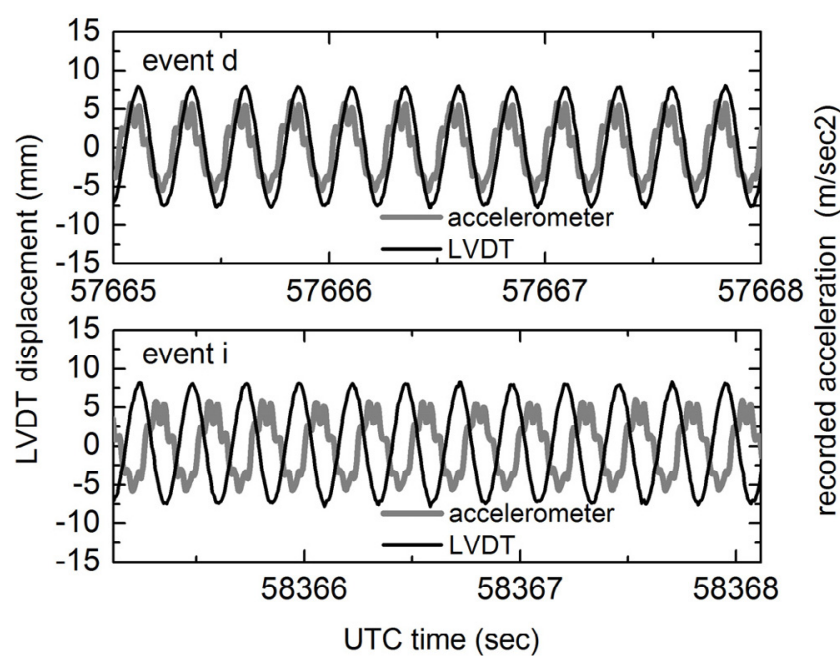

Fig. 4. Detailed view of two oscillation events (events d and i shown in Fig. 3) with same frequency $(4 \mathrm{~Hz})$ and amplitude $( \pm 8 \mathrm{~mm})$, separated by an interval of approximately $11 \mathrm{~min}-$ utes. Phase shifts between displacement (from LVDT) and acceleration differ from the theoretical value of $180^{\circ}$ and vary even between successive experiments.

the accelerometer data. A representative diagram is shown in Figure 5. A narrow spectral peak (consistent with the input frequency of the motor forcing the oscillator) revealed that the spectra were not characterized by spectral leakage; hence the oscillations were essentially harmonic.

At a second step, we computed the differences in phases derived from FFT between accelerometer and LVDT for each of the 86 events. We then formed a data base containing these differences, as well as the other characteristics of the oscillations (event ID, reference and oscillation amplitude).

Finally, phase differences $\varphi$ were transformed into time delays $t_{d}$ as a function of the oscillation frequency $f$, known from the motor input and identified in the LVDT and accelerometer spectra, using the formula:

$$
t_{d}=\frac{\varphi}{\pi f}
$$

A summary of results are shown in the composite Figures 6 and 7. Results are shown separately for days 1 and 2 and for days 3 and 4, because different LVDT were used (see Sect. 2.2). In these figures phase differences and time delays are shown as a function of the peak acceleration and of the oscillation frequency, respectively.

\section{Discussion}

Figure 6 reveals no correlation between phase changes and peak accelerations or oscillation frequencies for both LVDT instruments used.

A similar lack of correlation appears to exist between time delays and peak accelerations or oscillation frequencies (Fig. 7). In this last case, according to equation (4) a certain delay is indeed expected, and this is computed
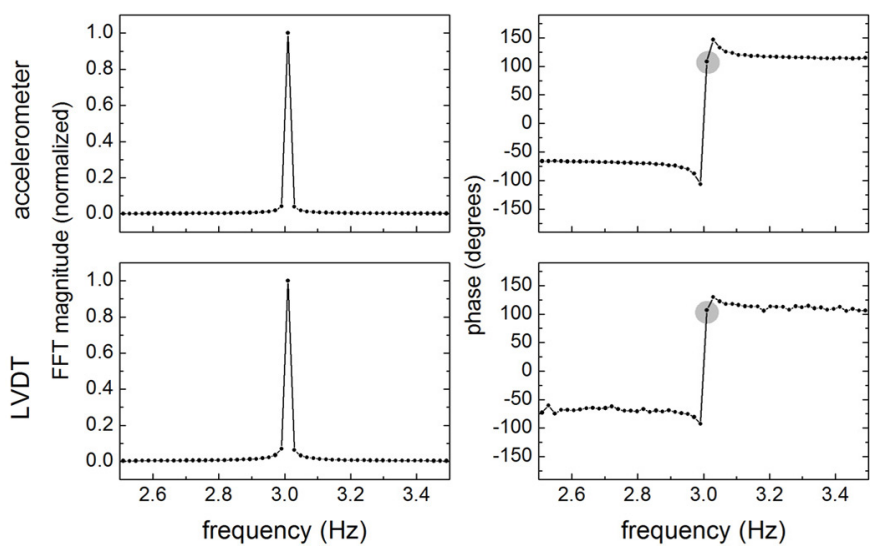

Fig. 5. Typical FFT results for a harmonic oscillation with $3 \mathrm{~Hz}$ frequency. The spectral peak is practically the same for the accelerometer and the LVDT, while the two time-series present almost the same phase (marked with a gray circle) for the peak frequency.
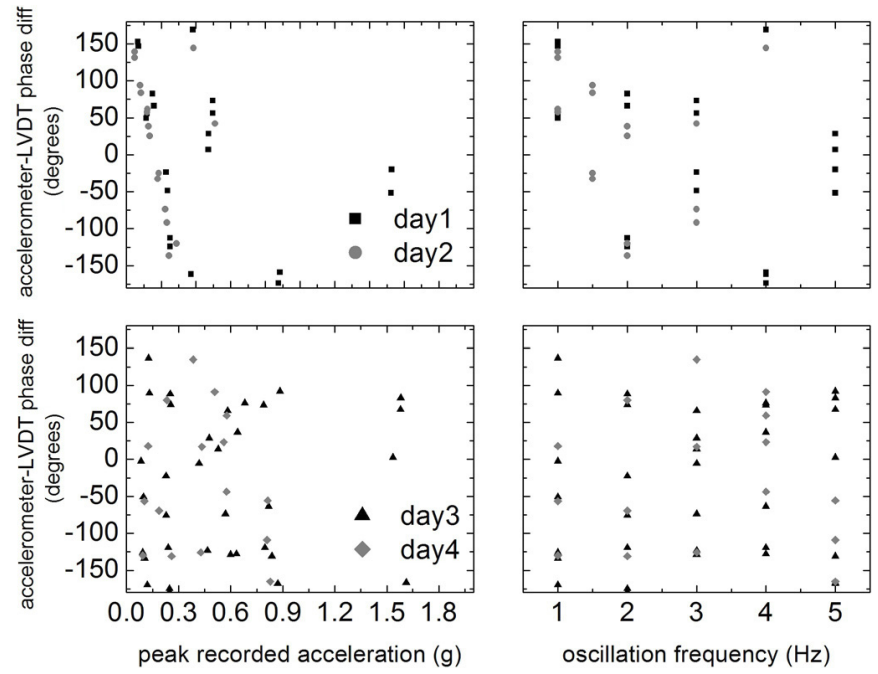

Fig. 6. Phase difference (in degrees) between peak recorded accelerations and displacements measured by the LVDT. In days 1, 2, 3 and 4 different phases can be identified between displacement and acceleration, even for oscillations with same oscillation frequency and acceleration.

and plotted in Figure 6 . There is, however, evidence, that the observed time delay is decreasing with increasing oscillation frequency, as predicted by equation (4).

For the majority of the experiments (especially for oscillation frequencies $>1 \mathrm{~Hz}$ ) the time delay is ranging between approximately 0.2 to $-0.2 \mathrm{~s}$.

Figures 6 and 7 tend to indicate that the amplitude of the phase difference/time delay between recorded acceleration and displacement does not follow a specific pattern, but has a rather random distribution. Still, a decrease in the time delay as a function of the oscillation frequency is induced as a consequence of equation (4). These results derive from observations with different LVDT sensors and for a wide range of phase differences for the same acceleration or oscillation frequency. In addition, this result 

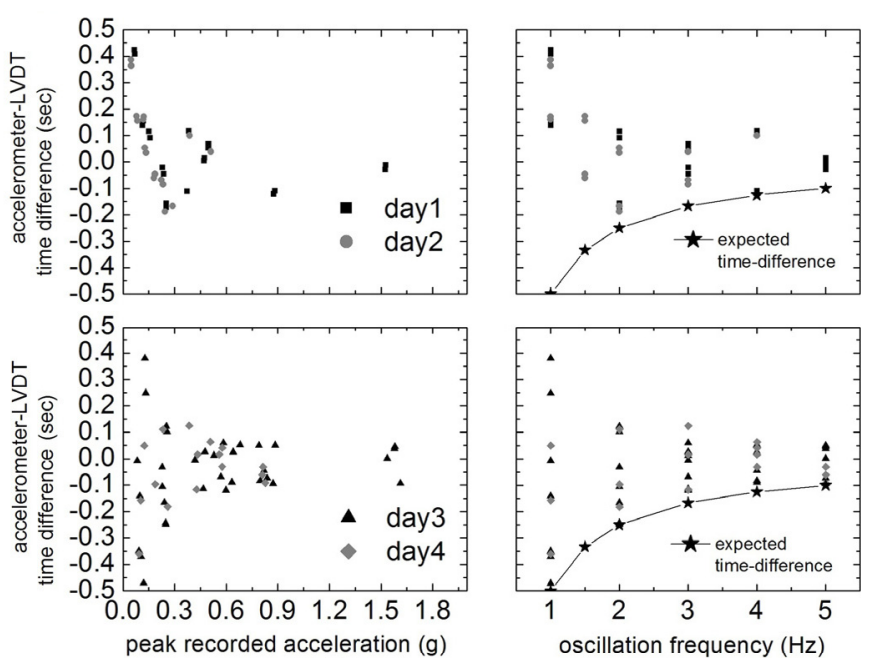

Fig. 7. Same as Figure 4 with phase shift expressed as time delay (in seconds). The curve of expected time-difference is derived from equation (4).

is obtained from experiments in different days, with rebooted instruments, and in successive experiments (see Figs. 2 and 3 ).

The question arising is whether these results are due to malfunction of accelerometers or LVDT. As indicated by Figure 8, a comparison of simultaneous recordings of accelerometer, LVDT and GPS indicates no phase error (delay) between the last two independent sensors, and hence a phase delay seems to characterize only accelerometers.

The experiments discussed were based on a specific type of high-quality force-balance accelerometer, accurate for low-frequencies such as those used in applications like civil engineering and seismology. Still, other experiments with other high-quality accelerometers, not discussed in this paper, lead to the same conclusion, and in fact have inspired this study. Hence, our conclusion is valid for a wide range of sensors.

These results are likely to suggest that (random) phase delays is another type of dynamic error affecting accelerometer measurements, and this error seems rather independent from the characteristics of the measured dynamic motion. Effects like random or systematic phase drifts (commonly referred to as phase jitter or phase perturbations) are known to affect dynamic measurements and oscillator-based systems (clock oscillators, measuring devices, etc.) while there exist several methods for modeling and filtering such effects [23,24].

Analyzed experiments indicate that there is strong indication that phase shift errors mainly affect the accelerometer system while LVDT and GPS seem free from it.

The reasons for the observed time shift between accelerometer and LVDT measurements are not clear. The use of similar recorders with dedicated GPS timing in the experiments discussed, makes rather unlikely the possibility this error to be related to the recording instruments, and a future study will be devoted to the analysis of signals using a common recording and timing platform.

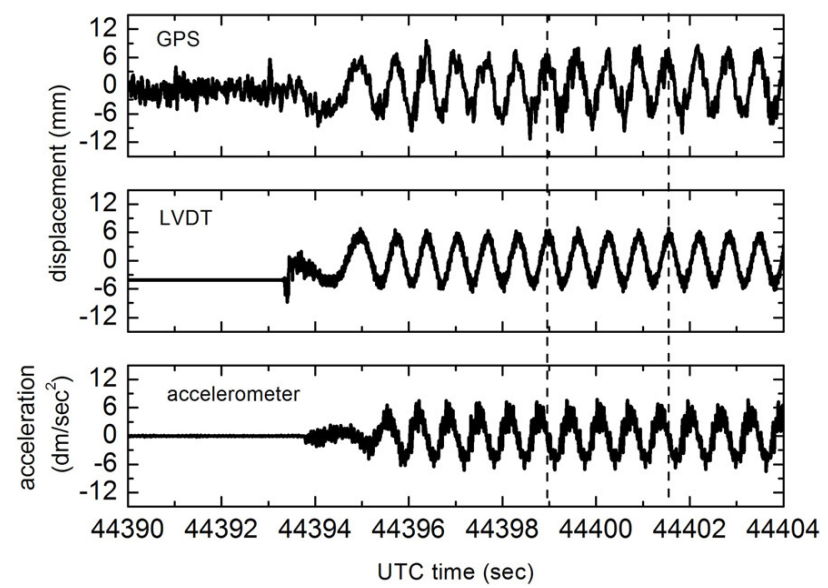

Fig. 8. Displacements measured by the LVDT and the GPS along with recorded accelerations. Dashed lines mark two moments in which the LVDT and GPS simultaneously present maximum displacement (hence no indication of phase between GPS and LVDT) while the accelerometer value does not correspond to a minimum as implied by equation (3) in Section 2 .

Still, two open questions remain: (a) how the phase shift, detected in single frequency harmonic oscilaltions, affects complicated signals simultaneously containing more than one significant frequencies (for example measurements of the dynamic response of a structure with high order natural frequencies) and (b) how the instrument drift affects the phase delay error for long intervals of oscillation (hours, etc.).

\section{Implications for sensor fusion}

The identification of a rather random phase shift in accelerometers is very important, for this type of instruments, and especially lower quality MEM's, are widely used in various applications in different levels of fusion of various types of sensors. Such applications include seismology [25,26], navigation [5] including robot control, mobile mapping and airborne laser altimetry [27], Structural Health Monitoring (SHM) [28, 29], etc. The identification of this type of dynamic error may represent a source of significant bias in the results of such instruments, and a possible limitation for their use.

\section{Sensor syncronization}

On the basis of the experimental results analyzed in the present study, a methodology for the synchronization of the accelerometer and the LVDT (or any other displacement-measuring sensor) can be proposed. This methodology consists of three steps (see Fig. 9):

(i) At a first step, using spectral analysis the phase $\varphi_{\alpha}$ of the accelerometer and $\varphi_{d}$ of the LVDT time-series are computed and their difference

$$
\varphi=\varphi_{a}-\varphi_{d}
$$

is calculated. 


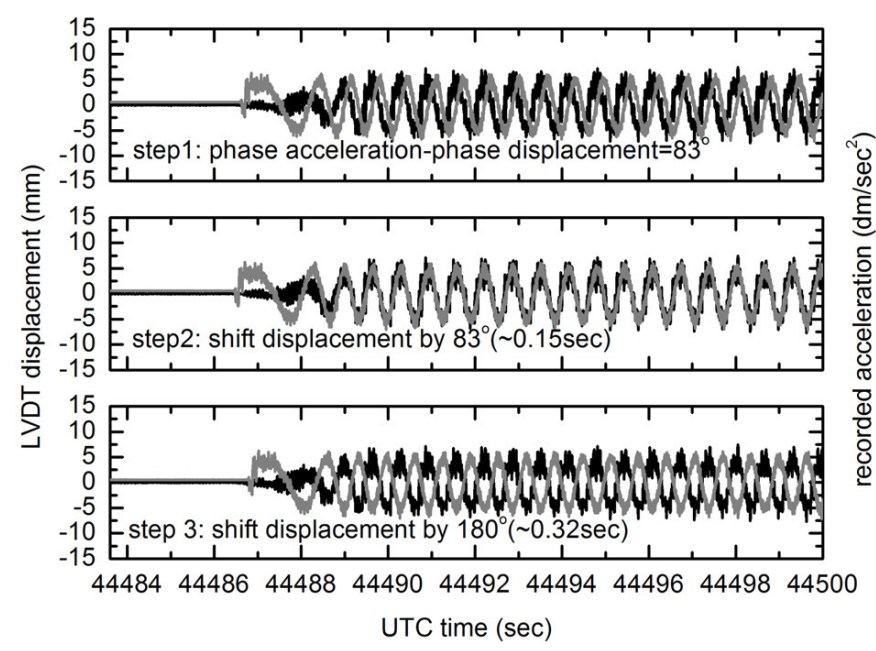

Fig. 9. A three-step procedure for syncronization of acceleration (accelerometer) and displacement (LVDT) measurements. The harmonic oscillation shown has amplitude $\pm 5 \mathrm{~mm}$ and frequency $1.5 \mathrm{~Hz}$.

(ii) The phase difference $\varphi$ is used to produce a first shift in the time-series of acceleration. This shift is imposed simply by applying equation (4) in the time stamping of the accelerometer measurements.

(iii) At a third step, a second time shift of $180^{\circ}$ ( $\pi$ radians) is introduced in order to produce the phase implied by equation (3) is introduced.

Figure 9 highlights this approach for two time series shown superimposed in the upper diagram. A phase difference of $83^{\circ}$ was computed according to equation (5) and this parameter was used to produce the shift shown in the middle diagram. In the lower diagram, an additional offset of $180^{\circ}$ was imposed on the time series of the middle diagram leading to two time series with a phase of $180^{\circ}$ between them, as is theoretically expected.

This process can be modified to accommodate corrections from other sensors measuring displacement (interferometers and possibly GPS or even microwave radars [30]), or can be modified to accommodate corrections from sensors with other theoretical offsets, such as high-accuracy velocimeters (offset of $90^{\circ}$, see Eq. (2)).

\section{Conclusions}

High-accuracy accelerometers were tested in a prototype experimental platform, compared with extensiometers (LVDT) and ultra-high frequency GPS, and were found to suffer from a rather random phase error. These experiemnts confirm the hypothesis of a phase error in accelerometers, derived on theoretical grounds [8] and indicate that such errors should be controlled and minimized in various combinations of sensors. A simple method to minimize this error has been proposed.
Acknowledgements. We thank George Chatzigiannelis and Argyris Georgakopoulos of EUROTECH Ltd for their help with the LVDT and accelerometer setup. The personnel of the Machine Shop of the Dept. of Physics of the Patras University, George Hatzipanayiotou and Gerasimos Diamantis made possible the design and construction of the experimental device. The present paper is a contribution to the Karatheodori 2009 Research Program (program ID: C-898) of the Patras University.

\section{References}

1. M.G. Kogan, W.-Y. Kim, Y. Bock, A.W. Smyth, Load response on a large suspension bridge during the NYC marathon revealed by GPS and accelerometers, Seismol. Res. Lett. 79, 12-19 (2008)

2. S. Godha, M. Cannon, GPS/MEMS INS integrated system for navigation in urban areas, GPS Solutions 11, 193-203 (2007)

3. G.Y. Ojeda, P.T. Gayes, R.F. Van Dolah, W.C. Schwab, Spatially quantitative seafloor habitat mapping: example from the northern South Carolina inner continental shelf, Estuar. Coast. Shelf Sci. 59, 399-416 (2004)

4. T. Kingston, V. Gikas, C. Laflamme, C. Larouche, An integrated mobile mapping system for data acquisition and automated asset extraction, 5th Int. Symposium on Mobile Mapping Technology Conference, ISPRS, Padua, Italy, 2007

5. C. Toth, S.W. Shin, D.A. Grejner-Brzezinska, J.H. Kwon, On accurate time synchronization of multi-sensor mobile mapping systems, J. of Applied Geodesy 2, 159-166 (2008)

6. W. Ding, J. Wang, Y. Li, P. Mumford, C. Rizos, Time synchronization error and calibration in integrated GPS/INS systems, ETRI Journal 30, 59-67 (2008)

7. M. Lombardi, The use of GPS disciplined oscillators as primary frequency standards for calibration and metrology laboratories, J. Meas. Technol. 3, 56-65 (2008)

8. J.P. Hessling, A novel method of estimating dynamic measurement errors, Meas. Sci. Technol. 17, 2740-2750 (2006)

9. R. Clough, W. Penzien, Dynamics of Structures, 3rd edn. (McGraw-Hill International Editions, 1993)

10. P. Psimoulis, S. Pytharouli, D. Karambalis, S. Stiros, Potential of Global Positioning System (GPS) to measure frequencies of oscillations of engineering structures, J. Sound Vib. 318, 606-623 (2008)

11. V. Spyropoulos, Design and construction of a calibrated apparatus for production of vertical harmonic oscillations, Unpublished Diploma Thesis, Laboratory of Geodesy and Geodetic Applications, Dept. of Civil Engineering, Univeristy of Patras, Patras, 2012 (in Greek)

12. W.-S. Chan, Y.-L. Xu, X.-L. Ding, Y.-L. Xiong, W.-J. Dai, Assessment of dynamic measurement accuracy of GPS in three directions, J. Surv. Eng. 132, 108-177 (2006)

13. X. Meng, G. Roberts, A. Dodson, E. Cosser, J. Barnes, C. Rizos, Impact of GPS satellite and pseudolite geometry on structural deformation monitoring: analytical and empirical studies, Journal of Geodesy 77, 809-822 (2004)

14. T. Herring, GLOBK: Global Kalman filter VLBI and GPS Analysis Program, Version 10.0 (Massachusetts Institute of Technology, Cambridge, 2000) 
15. F. Moschas, S. Stiros, Measurement of the dynamic displacements and of the modal frequencies of a short-span pedestrian bridge using GPS and an accelerometer, Eng. Struct. 33, 10-17 (2011)

16. G. Roberts, X. Meng, A. Dodson, Integrating a global positioning system and accelerometers to monitor the deflection of Bridges, J. Surv. Eng. 130, 65 (2004)

17. P. Elosegui, J.L. Davis, D. Oberlander, R. Baena, G. Ekström, Accuracy of high-rate GPS for seismology, Geophys. Res. Lett. 33, L11308 (2006)

18. S. Han, C. Rizos, Multipath effects on GPS in mine environments, 10th International Congress of the International Society for Mine Surveying, Fremantle, Australia, 1997

19. E.D. Kaplan, C.J. Hegarty, Understanding GPS: Principles And Applications (Artech House, Boston, 2006)

20. F. Moschas, S. Stiros, Noise Characteristics of Shortduration, High Frequency GPS-records, Advanced Mathematical and Computational Tools in Metrology and Testing, Vol. 9, edited by F. Pavese, M. Bär, J.-R. Filtz, A.B. Forbes, L. Pendrill, H. Shirono, Series on Advances in Mathematics for Applied Sciences (World Scientific, Singapore, 2012), Vol. 84

21. S. Stiros, P. Psimoulis, E. Kokkinou, Errors introduced by fluctuations in the sampling rate of automatically recording instruments: experimental and theoretical approach, J. Surv. Eng. 134, 89-93 (2008)

22. S. Pytharouli, S. Stiros, Spectral analysis of unevenly spaced or discontinuous data using the "normperiod" code, Comput. Struct. 86, 190-196 (2008)
23. A. Demir, A. Mehrotra, J. Roychowdhury, Phase noise in oscillators: a unifying theory and numerical methods for characterization, IEEE Trans. Circuits Syst. I: Fundam. Theory Appl. 47, 655-674 (2000)

24. J.P. Hessling, A novel method of dynamic correction in the time domain, Measurement Sci. Technol. 19, 075101 (2008)

25. L. Ge, L. Dai, S. Han, C. Rizos, Y. Ishikawa, Y. Yoshida, GPS Seismometers: the Implementing Issues, 13th Int. Technical Meeting of the Satellite Division of The Institute of Navigation (ION GPS 2000), Salt Lake City, UT, 2000, pp. $75-83$

26. A. Holland, Earthquake Data Recorded by the MEMS Accelerometer: Field Testing in Idaho, Seismol. Res. Lett. 74, 20-26 (2003)

27. M. Cocard, A. Geiger, H.-G. Kahle, G. Veis, Airborne laser altimetry in the Ionian Sea, Greece, Global Planet, Change 34, 87-96 (2002)

28. J.P. Lynch, Y. Wang, K.J. Loh, J.-H. Yi, C.-B. Yun, Performance monitoring of the Geumdang Bridge using a dense network of high-resolution wireless sensors, Smart Mater. Struct. 15, 1561-1575 (2006)

29. J. Hwang, H. Yun, S.-K. Park, D. Lee, S. Hong, Optimal methods of rtk-gps/accelerometer integration to monitor the displacement of structures, Sensors 12, 1014-1034 (2012)

30. V. Gikas, Ambient vibration monitoring of slender structures by microwave interferometer remote sensing, J. of Applied Geodesy 6, 167-176 (2012) 